\title{
Application of the consciousness model involving thought-carrying particle (TCP), thought retaining particle (TRP) and thought force (TF) to explain the cause of radioactivity
}

\author{
Dhananjay Pal \\ Pharmacy College, Bengal School of Technology, Sugandha-Delhi Road, Chuchura, Dist.-Hooghly, West Bengal, INDIA \\ Email address: \\ dhananjay.pal123@gmail.com, paldhananjay46@yahoo.com
}

\section{To cite this article:}

Dhananjay Pal. Application of the Consciousness Model Involving Thought-Carrying Particle (TCP), Thought Retaining Particle (TRP) and Thought Force (TF) to Explain the Cause of Radioactivity. American Journal of Physics and Applications.

Vol. 1, No. 3, 2013, pp. 55-58. doi: 10.11648/j.ajpa.20130103.12

\begin{abstract}
A single field emerged at the origin of the universe, already containing within itself the blueprint of the physical universe. The primordial single field triggered the onset of the universe. Most physicists believe that a single super-force dominated the first instants of creation. Scientists have arrived at a simple but decisive conclusion that consciousness is very much a part of the universe, like other objects. Our consciousness model involving thought-carrying particle (TCP), thought retaining particle (TRP) and thought force $\left(\mathrm{T}_{\mathrm{F}}\right)$ signifies the existence of universal consciousness that exists along with the universe. This universal consciousness is a functional state of the universal mind. This universal mind (UM) is evolved at the Big Bang from void. The UM is constituted by these TCP and TRP in the inherent presence of thought force $\left(\mathrm{T}_{\mathrm{F}}\right)$. Thought force $\left(\mathrm{T}_{\mathrm{F}}\right)$ is an expression of universal consciousness. The Thought force $\left(\mathrm{T}_{\mathrm{F}}\right)$ being the primordial quantum field functions as the original super-force. $\mathrm{T}_{\mathrm{F}}$ being the original super-force functions as the origin of all the fundamental fields. TCP is the carrier of thought force $\left(\mathrm{T}_{\mathrm{F}}\right)$ that, in turn, appears to be the origin of all the fields. The quantized energy $\left(\varepsilon_{T}\right)$ of TCP is responsible to cause the universal consciousness as well as the cosmic microwave background radiation temperature. The individual consciousness owes its origin to the universal consciousness created by the same $\varepsilon_{T}$. The same $\varepsilon_{T}$ is the energy responsible for generating thought force $\left(\mathrm{T}_{\mathrm{F}}\right)$. $\mathrm{T}_{\mathrm{F}}$ being an expression of the universal consciousness is applicable to any inanimate object as well as to any biological system (having thinking ability). The $\mathrm{T}_{\mathrm{F}}$ exerts its functions both in vitro and in vivo. We showed the existence of thought force in microcosm $\left[\mathrm{T}_{\mathrm{F}}\right.$ (micro)] and thought force in macrocosm $\left[\mathrm{T}_{\mathrm{F}}(\mathrm{macro})\right]$. This $\mathrm{T}_{\mathrm{F}}$ (micro) is theoretically found to be stronger than the strong nuclear force. $\mathrm{T}_{\mathrm{F}}$ (macro) is theoretically found to be weaker even than the gravitational force. . These TCP, TRP, $\mathrm{T}_{\mathrm{F}}$ (micro), $\mathrm{T}_{\mathrm{F}}$ (macro), and the thought force ( $\mathrm{T}_{\mathrm{F}}$ ) in vitro and thought force $\left(\mathrm{T}_{\mathrm{F}}\right)$ in vivo are found to play significant roles to explain the cause of radioactivity.
\end{abstract}

Keywords: Universal Mind (UM), Thought Force (TF), TF (Micro), TF (Macro), Though-Carrying Particle (TCP), Thought Retaining Particle (TRP), Quantized Energy $\left(\varepsilon_{T}\right)$ of TCP

\section{Introduction}

Consciousness model of Pal et al [1-3] involving TCP, TRP and thought force $\left(\mathrm{T}_{\mathrm{F}}\right)$ signifies the existence of universal consciousness that exists along with the universe. Pal et al [3] showed that the functional state of Universal Mind (UM) is the universal consciousness that exists along with the universe. Pal et al [3] explained that the UM is evolved at the Big Bang from the eternal Void. This Void, in turn, is the source of infinite energy. And this UM is a finer matter. The individual mind being a constituent of the UM is also a finer matter. The constituents of the UM and individual mind are the same. The ultimate constituents of matter and mind are the same as both mind and matter are aspects of one fundamental reality, which is called UM. The brain is the mediating link or interface between the individual mind and body.

Pal et al [3] explained that the constituents of the UM are the ultimate constituents of matter itself as everything in this universe is a manifestation of this UM. Pal et al [3] expressed that the UM is constituted by these TCP and TRP in the inherent presence of thought force $\left(\mathrm{T}_{\mathrm{F}}\right)$. Pal et al $[1,3]$ further explained that the ultimate constituents of matter and 
mind are these TCP and TRP in the inherent presence of thought force $\left(\mathrm{T}_{\mathrm{F}}\right)$ in vitro and thought force $\left(\mathrm{T}_{\mathrm{F}}\right)$ in vivo.

TCP cannot exist without TRP and vice versa. Many physicists believe that unifying all the forces, including gravity, into a single theory would require a phenomenon called super-symmetry. With super-symmetry, every fermion would have a boson twin, and vice-versa. TCP that behaves like boson should accompany its super-symmetrical partner TRP that functions like fermion in the generalized simpler way. Thus TCP like boson cannot have anti-particle. But TRP that functions like fermion should have its anti-particle and here it is shown as Anti-TRP. It is to be noted that these TCP and TRP function like wavicle: wave-particle duality.

\section{Thought Force}

Pal et al [1, 3] and Pal [4] explained the existence of the thought force $\left(\mathrm{T}_{\mathrm{F}}\right)$ that is an expression of the universal consciousness. Thought force $\left(\mathrm{T}_{\mathrm{F}}\right)$ is the primordial quantum field. Thought force $\left(\mathrm{T}_{\mathrm{F}}\right)$, an expression of the universal consciousness, is the primordial quantum field that, in turn, functions as the primary unified field. This $\mathrm{T}_{\mathrm{F}}$ being an expression of the universal consciousness is applicable to any inanimate object as well as to any biological system (having thinking ability). Thus the $\mathrm{T}_{\mathrm{F}}$ being an expression of the universal consciousness exerts its functions both in vitro and in vivo.

Physicists determined that underlying quantum fields give birth to elementary particles. Pal [4] expressed that the thought force $\left(\mathrm{T}_{\mathrm{F}}\right)$ is the primordial quantum field. Thought force $\left(\mathrm{T}_{\mathrm{F}}\right)$ being the primordial quantum field functions as the primary unified field. Thought force $\left(T_{F}\right)$ being the primordial quantum field gives birth to TRP that appears to be the origin of all the matter particles. TCP is the carrier of thought force $\left(\mathrm{T}_{\mathrm{F}}\right)$ that, in turn, appears to be the origin of all the fields. TCP thus appears to be the origin of all the field particles.

In a purpose to involve both the non-living and living systems of the world, Pal [4] has shown the existences of these TCP, TRP and thought force $\left(\mathrm{T}_{\mathrm{F}}\right)$ in vitro and thought force $\left(\mathrm{T}_{\mathrm{F}}\right)$ in vivo. Anyone can call this TCP by any other name, but as the highly developed living system will have to be evolved in the universe in the long run and as the thought of highly developed living system appears to be a kind of force to be called the thought force $\left(\mathrm{T}_{\mathrm{F}}\right)$ in vivo, we considered it is wise to call it as TCP. Further, as the universe exists along with the universal consciousness that, in turn, is created by the quantized energy $\left(\varepsilon_{T}\right)$ of TCP, we had to use the term TCP.

Pal (4) expressed that the non-living system of the world is governed by the thought force $\left(\mathrm{T}_{\mathrm{F}}\right)$ in vitro and this Thought force $\left(\mathrm{T}_{\mathrm{F}}\right)$ in vitro gives rise to $\mathrm{T}_{\mathrm{F}}$ (micro), $\mathrm{SNF}$, EMF, WNF, GF and $\mathrm{T}_{\mathrm{F}}$ (macro)

where $\mathrm{T}_{\mathrm{F}}$ (micro) $=$ Thought force in microcosm, $\mathrm{SNF}=$ Strong nuclear force, $\mathrm{EMF}=$ Electromagnetic force, $\mathrm{WNF}=$ Weak nuclear force, $\mathrm{GF}=$ Gravitational force and $\mathrm{T}_{\mathrm{F}}(\mathrm{ma}-$ cro) $=$ Thought force in macrocosm. It is to be noted here that $\mathrm{T}_{\mathrm{F}}$ (micro) is a stronger force than the $\mathrm{SNF}$ and $\mathrm{T}_{\mathrm{F}}$ (macro) is a weaker force even than the GF.

Pal (4) also expressed that the living system of the world is governed by the thought force $\left(\mathrm{T}_{\mathrm{F}}\right)$ in vivo and this Thought force $\left(\mathrm{T}_{\mathrm{F}}\right)$ in vivo is a type of force that represents the biological 'thought' which is the action of mind. This 'thought' being a type of force controls the 'thought processes' involving the firing of neurons through the quantum mechanical activities of these TCP and TRP in the presence of consciousness. This consciousness, in turn, is the quantized energy $\left(\varepsilon_{T}\right)$ of TCP. The thought force $\left(\mathrm{T}_{\mathrm{F}}\right)$ in vivo is demonstrated in numerous experiments in which thought has an effect on a physical process (often known as mind over matter). This biological 'thought' is a type of force that can cause movement. Controlling movement through thought alone is observed in several experiments conducted by many scientists as indicated by Pal (4). These experiments thus signify the existence of thought force $\left(\mathrm{T}_{\mathrm{F}}\right)$ in vivo.

Pal [4] explained the existence of $\mathrm{T}_{\mathrm{F}}$ (micro) (= Thought force in microcosm). This $\mathrm{T}_{\mathrm{F}}$ (micro) is the strongest interaction (a new class of 'extra strong' interaction). It is stronger than SNF (Strong Nuclear Force).

Pal [4] has also shown the existence of $\mathrm{T}_{\mathrm{F}}$ (macro) (= Thought force in macrocosm). It is the "weakest force" which is much weaker even than the gravity.

\section{One Typical Application Is Shown Theoretically to Support the Existence of TCP and TRP Along with the $T_{F}$ (Micro) for Explaining the Cause of Radioactivity}

It is to be noted that these TCP and TRP are interchangeable at super-symmetry and they function like wavicle: wave-particle duality. Pal [4] expressed that both the TCP and TRP are interchangeable at 'super-symmetry' as they have the same quantized energy and as they behave like biophotons in animals and like simple photons in inanimate objects.

Basis of calculation: $\mathrm{M}_{\mathrm{UR}}=$ radiant mass of the universe. Pal et al [3] have shown that

$$
\begin{gathered}
\mathrm{M}_{\mathrm{UR}}=\text { radiant mass of the universe }=V_{p r} \cdot \rho_{r}= \\
1.16025 \times 10^{53} \mathrm{~g}
\end{gathered}
$$

where $V_{p r}=$ present volume of the universe $=\left(\frac{4}{3}\right) \pi\left(\frac{c}{H_{0}}\right)^{3}=1.367 \times 10^{88} \mathrm{~cm}^{3}$, $H_{0}=$ present value of Hubble's parameter = $2.023988 \times 10^{-19} \mathrm{sec}^{-1}$,

$\mathrm{c} \quad=$ free-space velocity of light $=3 \times 10^{10} \mathrm{~cm} / \mathrm{sec}$,

$c / H_{0}=\mathrm{L}$ (Hubble length) $=$ radius of the universe $=\mathrm{R}=$ 
$1.482 \times 10^{29} \mathrm{~cm}$

$\rho_{r}=$ density of the radiant energy according to Stephan-Boltzmann formula indicated by Gamow [5],

$=8.5 \times 10^{-36} T^{4} \mathrm{~g} / \mathrm{cm}^{3}$.

$$
\begin{gathered}
\mathcal{E}_{T}=\text { quantized energy of the TCP }= \\
\left(\frac{h c}{\lambda_{T}}\right)=\left(\frac{h^{3} c^{5} m}{V_{p r}}\right)^{1 / 4}=4.95 \times 10^{-16} \mathrm{erg}, \\
m_{T}=\text { quantized mass of the TCP }=\varepsilon_{T} / c^{2} \\
=\left(\frac{h^{3} m}{c^{3} V_{p r}}\right)^{1 / 4}=5.5 \times 10^{-37} \mathrm{~g},
\end{gathered}
$$

Pal et al [3] expressed that TCP or TRP may be mathematically allotted a mass $\left(m_{T}\right)$ equivalent to $4.831 \times 10^{-37} \mathrm{~g}$ to $5.5 \times 10^{-37} \mathrm{~g}$.

Pal et al [3] have also shown that

$$
\varepsilon_{T}=
$$

$4.384 \times 10^{-16} \mathrm{erg} \equiv 2.73 \times 10^{-4} \mathrm{eV} \equiv 2.73^{0} \mathrm{~K} \cong C M B R$ temperature $\equiv 2.725^{\circ} \mathrm{K}$

where $\varepsilon_{T}=$ quantized energy of TCP.

It is to be noted that

$1 \mathrm{erg} \cong 0.6241807 \times 10^{12} \mathrm{eV}$ and $10^{-4} \mathrm{eV} \cong 1^{0} \mathrm{~K}$ where $\mathrm{K}=$ Kelvin indicated by Weisskopf [6].

The essential conversion chart is as follows:

$1 \mathrm{eV} \cong 1.6021 \times 10^{-12} \mathrm{erg} \cong 1.6021 \times 10^{-19} \mathrm{~J}=1 / 8066 \lambda=$ $23,060 \mathrm{kcal} / \mathrm{mole}$

$1 \mathrm{erg} \cong 0.6241807 \times 10^{12} \mathrm{eV} \cong 0.6241807 \times 10^{6} \mathrm{MeV} \cong$ $0.6241807 \times 10^{3} \mathrm{GeV}$

$1 \mathrm{cal} \cong 4.186 \times 10^{7} \mathrm{erg} \cong 4.186 \mathrm{~J} \cong 2.61282 \times 10^{13} \mathrm{MeV}$

$1 \mathrm{erg} \cong 2.388915 \times 10^{-8} \mathrm{cal}$

$\boldsymbol{\varepsilon}_{T}=$ quantized energy of TCP $=$

$4.95 \times 10^{-16} \mathrm{erg} \cong 3.897 \times 10^{-4} \mathrm{eV} \cong 3.0897 \times 10^{-10} \mathrm{MeV} \cong$

$3.0897 \times 10^{-13} \mathrm{GeV} \cong 11.82513 \times 10^{-24} \mathrm{cal}$.

Accepting the spherical size of a nucleus to be $10^{-12} \mathrm{~cm}$, the nuclear volume is found to be $10^{-36} \mathrm{~cm}^{3}$ which can normally accommodate $4.20645 \times 10^{14}$ TRP and TCP $\cong 231.35475 \times 10^{-24} \mathrm{~g} \cong 0.20821927 \mathrm{erg} \cong 130 \mathrm{GeV}$ accepting the volume of a TCP or TRP

$$
\left(V_{T}\right)=(4 / 3) \pi\left(r_{T n}\right)^{3}=2.3773 \times 10^{-51} \mathrm{~cm}^{3}
$$

where $\quad V_{T}=$ Volume of a TCP or TRP,

$r_{T n}=$ radius of a TCP or TRP within a nucleon $=$ $8.28 \times 10^{-18} \mathrm{~cm}$.

Again,

$$
\rho_{T n}=\rho_{n}=m_{n} / V_{n}=m_{n} /(4 / 3) \pi\left(r_{n}\right)^{3}=0.2313535 \times 10^{15} \mathrm{~g} / \mathrm{cm}^{3}
$$

where $\quad \rho_{T n}=$ density, i.e., concentration of the TRP and TCP within the structure of a nucleon,

$$
\begin{aligned}
& \rho_{n}=\text { density of a nucleon }, \\
& m_{n}=\text { rest mass of a nucleon } \approx 1.675 \times 10^{-24} \mathrm{~g}, \\
& V_{n}=\text { volume of a nucleon }=7.24 \times 10^{-39} \mathrm{~cm}^{3}, \\
& r_{n}=\text { radius of a nucleon }=1.2 \times 10^{-13} \mathrm{~cm} .
\end{aligned}
$$

When $\rho_{T n}=\rho_{n}=0.2313535 \times 10^{15} \mathrm{~g} / \mathrm{cm}^{3}$,

$V_{T}=m_{T} / \rho_{T n}=m_{T} / \rho_{n}=(4 / 3) \pi\left(r_{T n}\right)^{3}=2.3773 \times 10^{-51} \mathrm{~cm}^{3}$

where $\quad V_{T}=$ Volume of a TCP or TRP,

$m_{T}=$ quantized mass of a TCP or TRP $\approx 5.5 \times 10^{-37} \mathrm{~g}$,

$r_{T n}=$ radius of a TCP or TRP within a nucleon.

Thus, the radius of a TCP or TRP within a nucleon $\left(r_{T n}\right)=8.28 \times 10^{-18} \mathrm{~cm}$.

The required 'field' (within $10^{-12} \mathrm{~cm}$ ) = $0.20821927 \mathrm{erg} / 10^{-12} \mathrm{~cm}=2.0821927 \times 10^{11}$ dyne.

Further, accepting the quantized energy $\left(\varepsilon_{T}\right)$ of TCP $=$ $\mathcal{E}_{T}=4.384 \times 10^{-16} \mathrm{erg}$, it can be shown that

$T_{F}($ micro $) \cong 10^{5} S N F \cong 10^{44} \mathrm{GF} \cong 52.94$ dyne $\cong 33.04 \times 10^{12} \mathrm{eV} \cong 33.04 \times 10^{3} \mathrm{GeV} \cong$

Strongest interaction (at $D_{i}=10^{-18} \mathrm{~cm}$, the theoretically calculated radius of a TCP or TRP within a nucleon) where $\mathrm{T}_{\mathrm{F}}($ micro $)=$ Thought force in microcosm, $\mathrm{SNF}=$ strong nuclear force, $\mathrm{GF}=$ gravitational force and $\mathrm{Di}=$ interacting distance.

When one considers the case of Bismuth having mass number 209, atomic number 83 and isotopic mass 208.9804 $\mathrm{u}$, it is found that

$$
\begin{aligned}
& \mathrm{Bi}\left(208.9804 \mathrm{u}=346.99105 \times 10^{-24} \mathrm{~g}\right) \text { represents } \\
& 6.3089281 \times 10^{14} \text { TRPorTCP } \cong 194.56075 \mathrm{GeV} \cong 0.31229194
\end{aligned}
$$

erg for which the required 'field' (within $10^{-12} \mathrm{~cm}$ ) is $3.1229194 \times 10^{11}$ dyne.

Thus $\quad 6.3089281 \times 10^{14}$ TRPorTCP $\quad(\cong 195 \mathrm{GeV})$ would occupy a volume equivalent to

$6.3089281 \times 10^{14} \times 2.3773 \times 10^{-51} \mathrm{~cm}^{3}=1.4998214 \times 10^{-36} \mathrm{~cm}^{3}$.

This volume is found to be nearly $150 \%$ of the normal nuclear volume and would represent a 'field' of $3.1229194 \times 10^{11}$ dyne within $10^{-12} \mathrm{~cm}$.

If the number of TRP and TCP exceeds $6.3089281 \times 10^{14}$ to constitute any atomic structure, then that specific atom would exhibit radioactivity. Because the nuclear volume would then cross the "critical size" where the force of Coulomb repulsion would be greater than the attractive force between the nucleons to hold them together 
within this nucleus and this atom would then be found to have a $\mathrm{n} / \mathrm{p}$ ratio $>1.5$. And further, it would then have a mass equivalent of $195 \mathrm{GeV}$ (or $>195 \mathrm{GeV}$ ) which is much greater than $100 \mathrm{GeV}$, the mass equivalent of intermediate boson $\left(\mathrm{W}^{+}, \mathrm{W}^{-}\right.$and $\left.\mathrm{Z}^{0}\right)$ responsible for mediating radioactivity.

\section{Conclusion}

The consciousness model involving TCP, TRP and thought force $\left(\mathrm{T}_{\mathrm{F}}\right)$ along with the $\mathrm{T}_{\mathrm{F}}$ (micro) can explain the cause of radioactivity. It is already shown that if the number of TRP and TCP to constitute any atomic structure exceeds $6.3089281 \times 10^{14}$, then that specific atom would exhibit radioactivity due to the fact that this atom would then be found to have a $\mathrm{n} / \mathrm{p}$ ratio $>1.5$.

\section{References}

[1] D. Pal and A.U. De, Physics of consciousness and its model may provide guidelines to solve many scientific problems. Neuroquantology 1: 17-28(2004)

[2] D. Pal and A.U. De, Consciousness model: Significance of thought-carrying particles and thought-retaining particles in quantum measurement as well as cognitive problem. NeuroQuantology 2: 115-116 (2005)

[3] D. Pal and A.U. De, The cosmic microwave background radiation temperature signifying the existence of the thought-carrying particle, thought retaining particle and thought force. NeuroQuantology 10: Issue 3; $428-442$ (September 2012)

[4] D. Pal, Existence of thought force and its characteristics. Communicated to American Journal of Modern Physics (AJMP) (2013)

[5] G. Gamow, The Great Expansion. The World of Physics. (Simon and Schuster, 1230 Avenue of Americas, New York 10020) 3: 271-272 (1987)

[6] V. F. Weisskopf, The Origin of the Universe, The World of Physics, (Simon and Schuster, 1230 Avenue of Americas, New York 10020), 3: 10 (1987) 